\title{
Estudo das propriedades elétricas e térmicas de compósitos nanoestruturados de poli(sulfeto de fenileno) reforçados com nanotubos de carbono
}

\section{Electrical and Thermal study of carbon nanotubes reinforced poly (phenylene sulfide) nanostructured composites}

\author{
Bruno Ribeiro ${ }^{1 *}$, Edson Cocchieri Botelho ${ }^{1}$ e Michelle Leali Costa ${ }^{1}$ \\ ${ }^{1}$ Departamento de Materiais e Tecnologia, Universidade Estadual Paulista \\ "Júlio de Mesquita Filho" - UNESP, Guaratinguetá, SP, Brasil \\ *dorado.bruno@gmail.com
}

\begin{abstract}
Resumo
Neste trabalho o comportamento de cristalização e a condutividade elétrica de compósitos nanoestruturados de poli(sulfeto de fenileno) reforçado com nanotubos de carbono de paredes múltiplas obtidos através da técnica de mistura em fusão foram estudados. A incorporação do nanoreforço na matriz polimérica foi responsável por um aumento da cristalinidade devido ao fenômeno de nucleação heterogênea. A condutividade elétrica do PPS apresentou um aumento de 11 ordens de magnitude quando 2,0 m/m\% de MWCNT foram adicionados a matriz polimérica. Além disso, o limite de percolação elétrica encontrado para este sistema foi de $1,4 \mathrm{~m} / \mathrm{m} \%$ de MWCNT, revelando a formação de uma rede condutiva tridimensional no interior da matriz polimérica.
\end{abstract}

Palavras-chave: PPS, MWCNT, cristalização, propriedades elétricas, limite de percolação elétrica.

\section{Abstract}

In this work, the crystallization behavior and electrical conductivity of multiwalled carbon nanotubes reinforced poly (phenylene sulfide) nanostructured composites obtained by melt mixing were investigated. The incorporation of nanofiller in polymeric matrix was responsible for an increase in crystallinity due heterogeneous nucleation phenomenon. The electrical conductivity of PPS showed an increase by 11 orders of magnitude when $2.0 \mathrm{wt} \%$ of MWCNT was considered. Moreover, the electrical percolation threshold found on this system was $1.4 \mathrm{wt} \%$, suggesting the formation of three-dimensional conductive network within the polymeric matrix.

Keywords: PPS, MWCNT, crystallization, electrical properties, electrical percolation threshold.

\section{Introdução}

Os compósitos poliméricos condutores de eletricidade (CPCE) vêm recebendo grande destaque no meio científico/ acadêmico durante a última década devido principalmente as suas propriedades físicas e a processabilidade dos polímeros isolantes associada ao comportamento elétrico, óptico e magnético dos materiais condutores. Essa união de propriedades possibilita a estes materiais o uso em aplicações como aquecedores auto reguláveis, blindagem eletromagnética, dissipação de carga estática, biosensores, entre outras ${ }^{[1-4]}$.

Dentro deste conceito, os polímeros reforçados com nanotubos de carbono são considerados uma excelente alternativa, visto que sua obtenção pode resultar em materiais com diferentes níveis de condutividade elétrica, dando maior flexibilidade para o processamento de dispositivos eletrônicos com propriedades específicas. Entre os principais aspectos que devem ser considerados durante sua obtenção são a razão de aspecto destas nanopartículas, bem como sua distribuição e dispersão em diversas matrizes poliméricas ${ }^{[5,6]}$.

A quantidade de nanopartículas incorporadas na matriz desempenha um papel fundamental na obtenção dos CPCE. O mecanismo de condutividade destes materiais pode ser explicado devido a formação de uma rede tridimensional de CNTs no interior da matriz polimérica, sendo esta fortemente dependente da distribuição e da dispersão do nanoreforço. Quando a concentração de nanopartículas atinge um nível crítico, a condutividade elétrica da matriz aumenta de forma inesperada em um fenômeno conhecido como limite de percolação elétrica. Após este aumento 
abrupto na condutividade elétrica, a mesma apresentará aumentos modestos conforme o aumento do aditivo condutor no interior da matriz polimérica ${ }^{[6-8]}$

A condutividade elétrica dos CPCE vem sendo amplamente estudada com intuito de se obter materiais com propriedades elétricas avançadas. A escolha da matriz polimérica empregada, o método de obtenção dos compósitos nanoestruturados, a razão de aspecto dos CNTs e a dispersão/distribuição do reforço na matriz são fatores determinantes na obtenção dos $\mathrm{CPCE}{ }^{[6,9,10]}$.

A escolha da matriz polimérica afeta diretamente as propriedades elétricas dos CPCE. Diversos estudos tem reportado que o limite de percolação elétrica é maior em matrizes termoplásticas do que nos tradicionais sistemas termorrígidos. Este comportamento pode ser explicado devido a dois fatores: o primeiro é que as matrizes semicristalinas apresentam uma separação de fases induzida pelo processo de cristalização, ao passo que, o segundo está associado a rejeição dos nanotubos pelo avanço da fase cristalina ${ }^{[11-13]}$.

O método de obtenção empregado também afeta as propriedades elétricas do produto final. Os CPCE obtidos através do método de mistura em solução apresentam facilmente índices de percolação por volta de $0,2-1,0 \mathrm{~m} / \mathrm{m} \%$, enquanto que, os obtidos pelo método de mistura em fusão ocorrem por volta de $2,0-10,0 \mathrm{~m} / \mathrm{m} \%$. Baixos valores no limite de percolação elétrica podem ocorrer devido a estruturas cineticamente estáveis que são formadas pela boa dispersão do reforço, ao passo que, altos índices de percolação estão associados ao elevado grau de alinhamento das nanopartículas, ou pelo revestimento polimérico sobre os CNTs, limitando o contato entre os tubos. Além disso, o aumento da concentração dos CNTs na matriz polimérica durante a sua fusão provoca um grande aumento na viscosidade do sistema, afetando o processo de dispersão das nanopartículas, e consequentemente, elevando o limite de percolação elétrica ${ }^{[14,15]}$.

A razão de aspecto dos CNTs é outro fator que influencia diretamente nas propriedades elétricas do compósito obtido. Estudos anteriores ${ }^{[1,10,16]}$ apontam que para uma mesma concentração dos componentes em uma mistura condutiva, uma redução no limite de percolação é esperado a medida que a razão de aspecto dos CNTs aumenta. Isso ocorre devido a maior probabilidade de contato entre os tubos, uma vez que CNTs que apresentam maior comprimento são mais propensos a formar uma rede condutiva no interior da matriz polimérica.

A formação de uma rede condutiva quando baixas concentrações de CNTs são utilizadas não depende somente de uma boa distribuição do nanoreforço, mas da ocorrência de boa dispersão das nanopartículas. Uma má dispersão das nanopartículas impede a formação de uma rede condutiva adequada, impossibilitando que o fenômeno de percolação ocorra. Por outro lado, sistemas com elevada concentração de CNT dispersos estão aptos a formar uma rede condutiva no interior da matriz polimérica, mesmo com a existência de aglomerados. Neste caso, o fenômeno de percolação ocorrerá através das regiões aglomeradas do reforço, resultando em um aumento da condutividade elétrica do compósito, mesmo que uma boa dispersão não seja obtida ${ }^{[17-20]}$.

O fenômeno de cristalização é de grande importância na obtenção de compósitos poliméricos nanoestruturados, especialmente para a análise e operações de processamento. Portanto, o processo de cristalização dos compósitos poliméricos reforçados com CNTs deve ser analisado de modo a entender o potencial deste nanoreforço na aplicação em matrizes termoplásticas ${ }^{[21-23]}$. Estudos anteriores ${ }^{[24,25]}$ tem reportado que os CNTs podem atuar como agentes nucleadores durante o processo de cristalização. Mesmo que haja opiniões divergentes na literatura, se os nanotubos são cobertos por camadas cristalinas ou se estão localizados nas regiões amorfas dos polímeros semicristalinos, é aceito que a condutividade elétrica de compósitos nanoestruturados é fortemente influenciada pela formação da rede cristalina. $\mathrm{Se}$ as camadas cristalinas se formarem ao redor dos CNTs, cujos tamanhos dependem da quantidade do reforço e do seu estado de dispersão, elevados níveis de capacitância estarão associados as zonas de contato entre os tubos, reduzindo assim a condutividade elétrica do sistema ${ }^{[18]}$.

O presente trabalho tem como objetivo a avaliação das propriedades elétricas e do processo de cristalização dos compósitos nanoestruturados de PPS/MWCNT obtidos através da técnica de mistura em fusão, visando aplicações avançadas.

\section{Experimental}

\subsection{Materiais utilizados}

O poli(sulfeto de fenileno) (PPS) foi fornecido pela empresa Ticona (sob o nome comercial de Fortron 0205P4) na forma de grânulos. Os nanotubos de carbono de paredes múltiplas (MWCNTs), utilizados neste trabalho, foram produzidos pela técnica de deposição química de vapor (CVD) e fornecidos pela empresa Bayer, codificado como Baytubes C 150 P. Estes são caracterizados por apresentarem um diâmetro médio de suas paredes entre 13-16 nm, número de paredes entre 3-15 e massa específica entre $140-160 \mathrm{~kg} / \mathrm{m}^{3}$.

\subsection{Obtenção dos compósitos nanoestruturados de PPS/MWCNT}

Os compósitos nanoestruturados de PPS/MWCNT foram processados por meio da técnica de mistura em fusão em uma micro extrusora de rosca dupla (DSM Xplore), a $300{ }^{\circ} \mathrm{C}$, velocidade de rotação de $300 \mathrm{rpm}$ e tempo de residência de 1 minuto. Ao fim do processo, amostras com $0,5,1,0,2,0$ e 4,0\% em massa de MWCNT foram obtidas. Com o intuito de ter um melhor entendimento do limite de percolação elétrica, amostras com 3,0, 6,0 e 8,0 m/m\% de MWCNT foram preparadas. Amostras de PPS foram preparadas utilizando o mesmo procedimento descrito acima com o objetivo de padronizar os ensaios térmicos e elétricos. Após a extrusão todas as amostras foram secas em uma estufa a vácuo a $120^{\circ} \mathrm{C}$ por 3 horas.

Após o processamento por fusão, os compósitos obtidos foram moldados por compressão utilizando uma prensa hidráulica (Carver Hot Press, modelo 3725), a temperatura 
de $300{ }^{\circ} \mathrm{C}$ por $1 \mathrm{~min}$, a uma pressão de $10 \mathrm{MPa}$. Foram obtidos filmes com geometria retangular e espessura de aproximadamente $0,5 \mathrm{~mm}$, visando a caracterização elétrica do material.

\subsection{Calorimetria exploratória diferencial (DSC)}

A cristalização dos compósitos nanoestruturados foi estudada com auxílio da calorimetria exploratória diferencial (DSC) sob condições dinâmicas em um equipamento SII Nanotechnology SEIKO Modelo 6220, previamente calibrado com índio e zinco, sob fluxo constante de nitrogênio $(20 \mathrm{~mL} / \mathrm{min})$ a taxa de aquecimento/ resfriamento de $10^{\circ} \mathrm{C} / \mathrm{min}$. O ciclo térmico utilizado neste experimento foi iniciado com o aquecimento das amostras de $150^{\circ} \mathrm{C}$ até $340^{\circ} \mathrm{C}$, permanecendo 5 minutos nesta temperatura, para permitir a fusão completa da matriz, evitando assim, que cristais remanescentes atuassem como sementes durante a cristalização e posterior resfriamento de $340^{\circ} \mathrm{C}$ até $150^{\circ} \mathrm{C}$. A massa utilizada das amostras foi ajustada de modo a não provocar vazamento das mesmas no porta-amostra de alumínio hermeticamente fechado e possibilitar a obtenção de dados reprodutivos variando, assim, de 10 a $15 \mathrm{mg}$.

\subsection{Propriedades elétricas}

As análises de condutividade elétrica foram realizadas através do método de 4 pontas (Jandel Multi Height Probe, RM 3000), de acordo com a norma ASTM D-257 a temperatura ambiente. Para estas medidas, foram realizados 3 medidas em 5 corpos de prova retangulares $(30 \times 10 \times 0,5 \mathrm{~mm})$ obtidos através da moldagem por compressão.

\section{Resultados e Discussões}

\subsection{Cristalização de compósitos nanoestruturados de PPS/MWCNT}

É aceito que o processo de cristalização desempenha um papel importante nas propriedades físicas e mecânicas dos polímeros semicristalinos. Portanto, a influência da adição de nanoreforços em matrizes termoplásticas deve ser investigada de modo a ter uma melhor compreensão das propriedades do produto final. A Figura 1 apresenta as curvas de cristalização obtidas por DSC para os compósitos nanoestruturados de PPS/MWCNT a taxa de resfriamento de $10^{\circ} \mathrm{C} / \mathrm{min}$. Como pode ser observado a temperatura de pico de cristalização $\left(\mathrm{T}_{\mathrm{C}}\right)$ correspondente ao PPS puro foi de $224,1^{\circ} \mathrm{C}$. No entanto, o efeito da adição de MWCNT no PPS foi evidente, deslocando os picos de cristalização para temperaturas mais altas e consequentemente, aumentando os valores de $\mathrm{T}_{\mathrm{C}}$ para os compósitos nanoestruturados. Como observado a partir da Tabela 1 a temperatura de cristalização da matriz polimérica variou significativamente, com um aumento de $16^{\circ} \mathrm{C}$ pela adição de apenas $0,5 \mathrm{~m} / \mathrm{m} \%$ do reforço nanoestruturado. Este comportamento é explicado pelo efeito de nucleação heterogênea dos MWCNT. Durante a fusão do matriz polimérica, seus segmentos macromoleculares podem se ligar facilmente a superfície rígida dos tubos, fazendo com que o processo de cristalização do PPS ocorra a temperaturas mais elevadas. Além disso, os picos de cristalização dos compósitos nanoestruturados são mais estreitos quando comparados ao PPS puro, sugerindo que o processo de cristalização ocorreu de maneira mais acelerada devido a introdução dos MWCNT. De acordo com a literatura ${ }^{[26]}$, a ocorrência de picos de cristalização mais

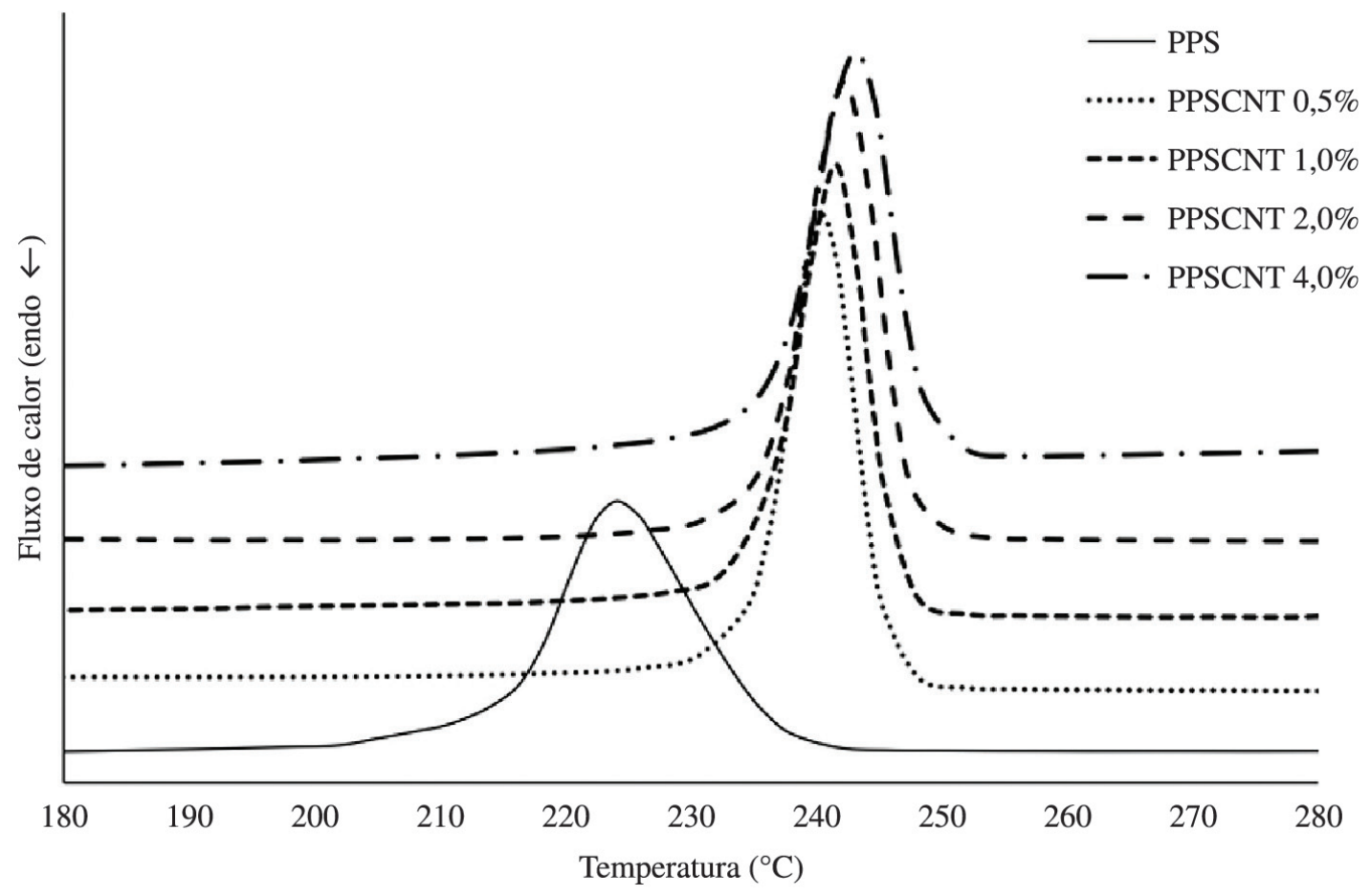

Figura 1. Curvas de DSC para os compósitos nanoestruturados de PPS/MWCNT a taxa de $10{ }^{\circ} \mathrm{C} / \mathrm{min}$. 
estreitos está associada a alta condutividade térmica dos MWCNTs quando comparados ao polímero puro, fazendo com que o calor seja melhor distribuído nos compósitos nanoestruturados de PPS/MWCNT.

Os valores de cristalinidade $\left(\mathrm{X}_{\mathrm{c}}\right)$ para o PPS e seus compósitos nanoestruturados foram calculados de acordo com a Equação 1:

$$
\chi_{c}=\frac{\Delta H_{m}}{\left[(1-\varphi) \cdot \Delta H_{m}^{0}\right]} \cdot 100
$$

Onde, $\Delta \mathrm{H}_{M}$ é a entalpia de fusão obtida para as amostras dos compósitos nanoestruturados, é a entalpia de fusão para o $\operatorname{PPS}^{[27]} 100 \%$ cristalino $(80 \mathrm{~J} / \mathrm{g}$ ) e é a concentração em massa do nanoreforço na matriz polimérica. Como pode ser observado a partir da Tabela 1, as amostras contendo até $1,0 \mathrm{~m} / \mathrm{m} \%$ de MWCNT apresentaram um aumento de cristalinidade, ao passo que, para os compósitos com concentração superior a $1,0 \mathrm{~m} / \mathrm{m} \%(2,0$ e $4,0 \mathrm{~m} / \mathrm{m} \%)$ foi verificado uma pequena redução na cristalinidade. Este comportamento pode ser explicado uma vez que o processo de cristalização em polímeros semicristalinos é controlado respectivamente pela nucleação e pelo crescimento do cristal. Neste caso, a adição de até $1,0 \mathrm{~m} / \mathrm{m} \%$ de MWCNT na matriz PPS resulta em um rápido aumento dos sítios de nucleação, na taxa de nucleação e na cristalinidade das amostras. Por outro lado, para concentrações acima de

Tabela 1. Parâmetros do processo de cristalização dos compósitos nanoestruturados de PPS/MWCNT.

\begin{tabular}{ccc}
\hline Amostras & $\mathbf{T}_{\mathbf{C}}\left({ }^{\circ} \mathbf{C}\right)$ & $\mathbf{X}_{\mathbf{C}}(\mathbf{\%})$ \\
\hline PPS & 224,1 & 50,1 \\
PPSCNT0,5\% & 240,5 & 53,7 \\
PPSCNT1,0\% & 241,5 & 54,5 \\
PPSCNT2,0\% & 242,2 & 53,9 \\
PPSCNT4,0\% & 243,9 & 52,8 \\
\hline
\end{tabular}

$1,0 \mathrm{~m} / \mathrm{m} \%$ a taxa de nucleação aumenta de maneira muito mais lenta. Além disso, a formação da rede de nanotubos no interior da matriz polimérica restringe o movimento das cadeias do PPS, impedindo que os cristais formados cresçam de forma adequada, reduzindo a cristalização do sistema. No entanto, como pode ser observado a partir da Tabela 1, a redução do grau de cristalinidade dos compósitos com concentração de nanoreforço superior a $1,0 \mathrm{~m} / \mathrm{m} \%$ não foi significativa, uma vez que os valores de $\mathrm{X}_{\mathrm{c}}$ para todas as amostras de PPS/MWCNT são de aproximadamente 54\%.

\subsection{Condutividade elétrica}

Devido ao seu elevado valor de resistividade elétrica $\left(\sim 10^{16} \Omega \mathrm{cm}\right)$ o PPS é classificado como um polímero isolante, limitando sua aplicação na indústria eletrônica. Materiais que apresentam condutividade elétrica superior a $10^{-8} \mathrm{~S} / \mathrm{cm}$ podem ser utilizados em aplicações de dissipação de carga eletrostática, ao passo que, aplicações voltadas a pintura eletrostática e proteção contra interferência eletromagnética requerem condutividades da ordem de $10^{-6}$ e $10^{-1} \mathrm{~S} / \mathrm{cm}$, respectivamente ${ }^{[28]}$. Portanto, a adição dos MWCNT no PPS visando a obtenção de compósitos nanoestruturados se faz necessária, de modo a ampliar a campo de aplicação desta matriz polimérica.

A Figura 2 apresenta a condutividade elétrica dos compósitos nanoestruturados de PPS/MWCNT a temperatura ambiente em função da concentração do nanoreforço. Como pode ser observado, a condutividade elétrica da matriz aumenta em função do aumento da concentração do aditivo condutor, apresentando um comportamento típico associado ao fenômeno de percolação. Para concentrações de até $1.0 \mathrm{~m} / \mathrm{m} \%$ os CPCE apresentam um comportamento similar ao PPS puro, mantendo-se como materiais isolantes $\left(\sigma<10^{-8} \mathrm{~S} / \mathrm{cm}\right)$. No entanto, os valores de condutividade elétrica sofrem um aumento significativo de 11 ordens de magnitude quando a concentração do nanoreforço varia entre 1 e $2 \mathrm{~m} / \mathrm{m} \%$.

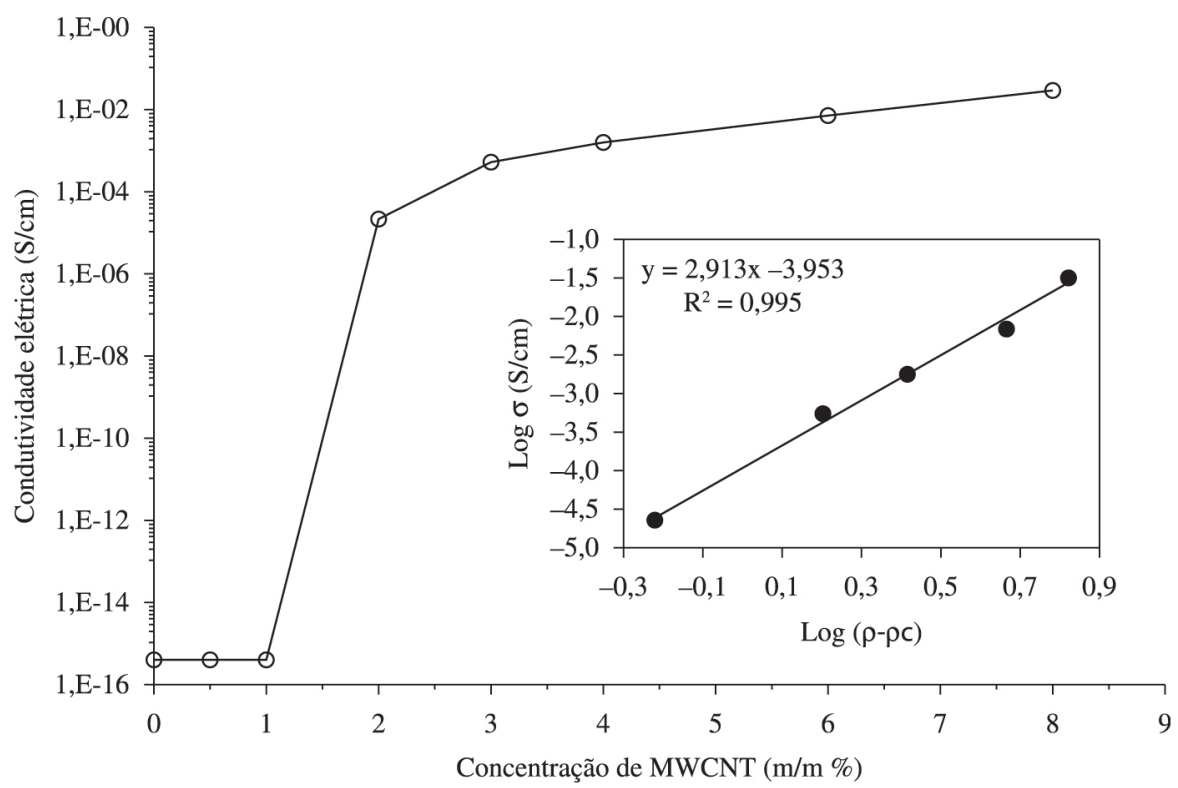

Figura 2. Condutividade elétrica em função da concentração do nanoreforço para os compósitos nanoestruturados de PPS/MWCNT. 
Este fenômeno está associado a ocorrência do limite de percolação elétrica, onde o material em estudo passa a apresentar o comportamento de um semicondutor. Aumentos posteriores na concentração dos MWCNTs geram aumentos modestos na condutividade elétrica dos CPCE. A Tabela 2 apresenta a evolução dos valores da condutividade elétrica em função da concentração do reforço para o compósito em estudo.

O limite de percolação elétrica dos $\operatorname{CPCE}\left(\rho_{C}\right)$ pode ser definido de acordo com a Equação 2, onde a condutividade elétrica do compósito $(\sigma)$ é relacionada com a concentração em massa dos MWCNT ${ }^{[29]}$ :

$$
\sigma=\sigma_{0}\left(\rho-\rho_{c}\right)^{t} \text { para } \rho>\rho_{c}
$$

Onde: $\sigma$ e $\sigma_{0}$ correspondem a condutividade do compósito e da fase condutora, respectivamente, $\rho$ é a concentração do componente condutivo e $t$ é o expoente crítico. A partir da inclinação da curva de $\log (\sigma)$ em função do $\log \left(\rho-\rho_{C}\right)$ pode-se determinar o valor do expoente crítico e da condutividade da fase condutora. Segundo a teoria da percolação, o expoente crítico $(\mathrm{t})$ pode ser definido como o número médio de contatos por partícula no limite de percolação. Para sistemas bidimensionais e tridimensionais, os valores de t podem variar de 1,1 a 1,3 e 1,6 a 2,0, respectivamente ${ }^{[1,30]}$. No entanto, valores entre 1 e 4 têm sido constantemente reportados na literatura, não havendo dependência quanto ao tipo de polímero empregado, método de obtenção utilizado, limiar de percolação, características dos CNTs (comprimento dos tubos, funcionalização, etc) ou valores máximos de condutividade elétrica ${ }^{[9]}$.

A Tabela 3 apresenta os valores dos parâmetros obtidos a partir da Equação 2. Como pode ser observado o limite de percolação elétrica e o expoente crítico encontrado para este estudo foram de $1.4 \mathrm{~m} / \mathrm{m} \%$ e 2,91, respectivamente. Estudos anteriores apontam que os valores de $\rho_{C}$ determinados para sistemas nanoestruturados de PPS/MWCNT foram iguais ou superiores ao reportado neste trabalho. Noll e Burkhart ${ }^{[31]}$ obtiveram valores ligeiramente inferiores $(\sim 1.0 \mathrm{~m} / \mathrm{m} \%)$, ao passo que Han et al. ${ }^{[32]}$ e Yang et al. ${ }^{[3]}$ determinaram, respectivamente, valores de percolação de $3.0 \mathrm{~m} / \mathrm{m} \%$ e $5.0 \mathrm{~m} / \mathrm{m} \%$. A partir dos dados citados acima, fica claro que a escolha da técnica de mistura em fusão foi adequada para

Tabela 2. Valores de condutividade elétrica em função da concentração do reforço para os compósitos nanoestruturados de PPS/MWCNT.

\begin{tabular}{cc}
\hline MWCNT $(\mathbf{m} / \mathbf{m} \%)$ & Condutividade elétrica $\mathbf{( S / c m )}$ \\
\hline 0 & $4,00 \times 10^{-16}$ \\
0,5 & $4,00 \times 10^{-16}$ \\
1,0 & $4,00 \times 10^{-16}$ \\
2,0 & $2,29 \times 10^{-5}$ \\
3,0 & $5,58 \times 10^{-4}$ \\
4,0 & $1,75 \times 10^{-3}$ \\
6,0 & $7,31 \times 10^{-3}$ \\
8,0 & $3,12 \times 10^{-2}$ \\
\hline
\end{tabular}

Tabela 3. Parâmetros elétricos obtidos para os compósitos nanoestruturados de PPS/MWCNT.

\begin{tabular}{ccc}
\hline $\boldsymbol{\sigma}_{\mathbf{0}}(\mathbf{S} / \mathbf{c m})$ & $\boldsymbol{\rho}(\mathbf{m} / \mathbf{m} \%)$ & $\mathbf{t}$ \\
\hline $1,10 \times 10^{-4}$ & 1,40 & 2,91 \\
\hline
\end{tabular}

a obtenção dos compósitos de PPS/MWCNT, promovendo a formação de uma rede tridimensional condutiva no interior da matriz polimérica e levando a um baixo índice de percolação elétrica. Além disso, foi observado que o valor de (t) encontrado neste estudo foi maior que o esperado, conforme apresentado na teoria da percolação descrita na Equação 2. Yang et al. ${ }^{[33]}$ e Jang et al. ${ }^{[34]}$ determinaram, respectivamente, valores de 3,6 e 3,3 para o expoente critico de compósitos nanoestruturados de PPS/MWCNT. Este comportamento pode ser explicado através do modelo de percolação de tunelamento ${ }^{[35]}$, onde as partículas condutoras estão dispersas de maneira não aleatória no sistema, ou ainda sob a forma de pequenos aglomerados na matriz polimérica. A aglomeração dos MWCNTs pode ter causado o contato entre os tubos, permitindo a transferência de carga entre as partículas de nanoreforço, o que resultou no alto valor de (t).

\section{Conclusões}

Compósitos nanoestruturados de PPS reforçados com MWCNT foram obtidos através da técnica de mistura em fusão, e os efeitos da presença do nanoreforço na cristalização e nas propriedades elétricas do material foram investigadas.

A incorporação dos MWCNT na matriz polimérica foi responsável pelo aumento da cristalização do sistema através da nucleação heterogênea, provocando um aumento nas temperaturas de pico e na taxa de cristalização dos compósitos nanoestruturados. No entanto, este efeito foi mais evidente em compósitos nanoestruturados de PPS/MWCNT com $0,5 \mathrm{~m} / \mathrm{m} \%$.

A adição de 2,0 m/m\% do nanoreforço foi responsável por a um aumento de 11 ordens de magnitude na condutividade elétrica dos compósitos nanoestruturados, de modo que o limite de percolação elétrica do material obtido neste estudo foi de $1,4 \mathrm{~m} / \mathrm{m} \%$. Este valor é em geral inferior aos anteriormente reportados na literatura, comprovando a formação de uma rede condutiva tridimensional no interior da matriz polimérica.

\section{Agradecimentos}

Os autores agradecem a FAPESP (projeto 2008/00171-8) e ao CNPq (474160/2010-6 e 303559/2012-8) pelo suporte financeiro, sem os quais a realização deste trabalho não seria possível.

\section{Referências}

1. Ramoa, S. D. A. S. (2011). Preparação e caracterização de compósitos de poliuretano termoplástico com negro de fumo condutor e nanotubos de carbono (Dissertação de mestrado). Universidade Federal de Santa Catarina, Florianópolis.

2. Rahmat, M., \& Hubert, P. (2011). Carbon nanotube-polymer interactions in nanocomposites: a review. Composites Science and Technology, 72(1), 72-84. http://dx.doi.org/10.1016/j. compscitech.2011.10.002.

3. Yang, J., Xu, T., Lu, A., Zhang, Q., Tan, H., \& Fu, Q. (2009). Preparation and properties of poly (p-phenylene sulfide)/ multiwall carbon nanotube composites obtained by melt 
compounding. Composites Science and Technology, 69(2), 147153. http://dx.doi.org/10.1016/j.compscitech.2008.08.030.

4. Spitalsky, Z., Tasis, D., Papagelis, K., \& Galiots, C. (2010). Carbon nanotube-polymer composites: Chemistry, processing, mechanical and electrical properties. Progress in Polymer Science, 35(3), 357-401. http://dx.doi.org/10.1016/j. progpolymsci.2009.09.003.

5. Castillo, F. Y., Socher, R., Krause, B., Headrick, R., Grady, B. P., Prada-Silvy, R., \& Pötschke, P. (2011). Electrical, mechanical, and glass transition behavior of polycarbonatebased nanocomposites with different multi-walled carbon nanotubes. Polymer, 52(17), 3835-3845. http://dx.doi. org/10.1016/j.polymer.2011.06.018.

6. Botelho, E. C. (2011). Processamento e caracterização de compósitos de resina fenólica com nanotubos de carbono com aplicações aeroespaciais (Tese de Livre-Docência). Universidade Estadual Paulista, Guaratinguetá.

7. Bose, S., Khare, R. A., \& Moldenaers, P. (2010). Assessing the strengths and weaknesses of various types of pre-treatments of carbon nanotubes on the properties of polymer/carbon nanotubes composites: A critical review. Polymer, 51(5), 975-993. http://dx.doi.org/10.1016/j.polymer.2010.01.044.

8. Wang, W., Lu, F., Veca, L. M., Meziani, M. J., Wang, X., Cao, L., Gu, L., \& Sun, Y. P. (2011). Carbon Nanotubes and Nanocomposites for Electrical and Thermal Applications. New York: John-Wiley. http://dx.doi.org/10.1002/9781119951438. eibc0320.

9. Grady, B. P. (2011). Carbon nanotube polymer composites: Manufacture, Properties, and Applications. New York: JohnWiley. http://dx.doi.org/10.1002/9781118084380.

10. Bauhofer, W., \& Kovacs, J. Z. (2009). A review and analysis of electrical percolation in carbon nanotube polymer composites. Composites Science and Technology, 69(10), 1486-1498. http://dx.doi.org/10.1016/j.compscitech.2008.06.018.

11. Abbasi, S., Derdouri, A., \& Carreau, P. J. (2011). Properties of microinjection molding of polymer multiwalled carbon nanotube conducting composites. Polymer Engineering and Science, 51(5), 992-1003. http://dx.doi.org/10.1002/ pen. 21904 .

12. Abbasi, S., Carreau, P. J., \& Derdouri, A. (2010). Flow induced orientation of multiwalled carbon nanotubes in polycarbonate nanocomposites: Rheology, conductivity and mechanical properties. Polymer, 51(4), 922-935. http://dx.doi. org/10.1016/j.polymer.2009.12.041.

13. Sumfleth, J., Prehn, K., Wichmann, M. H. G., Wedekind, S., \& Schulte, K. (2010). A comparative study of the electrical and mechanical properties of epoxy nanocomposites reinforced by CVD- and arc-grown multi-wall carbon nanotubes. Composites Science and Technology, 70(1), 173-180. http:// dx.doi.org/10.1016/j.compscitech.2009.10.007.

14. Bangarusampath, D. S., Ruckdäschel, H., Altstädt, V., Sandler, J. K. W., Garray, D., \& Shaffer, M. S. P. (2009). Rheology and properties of melt-processed poly(ether ether ketone)/ multi-wall carbon nanotube composites. Polymer, 50(24), 5803-5811. http://dx.doi.org/10.1016/j.polymer.2009.09.061.

15. Socher, R., Krause, B., Muller, M. T., Boldt, R., \& Pötschke, P. (2012). The influence of matrix viscosity on MWCNT dispersion and electrical properties in different thermoplastic nanocomposites. Polymer, 53(2), 495-504. http://dx.doi. org/10.1016/j.polymer.2011.12.019.

16. Bangarusampath, D. S., Ruckdäschel, H., Altstädt, V., Sandler, J. K. W., Garray, D., \& Shaffer, M. S. P. (2009). Rheological and electrical percolation in melt-processed poly(ether ether ketone)/multi-wall carbon nanotube composites. Chemical Physics Letters, 482(1-3), 105-109. http://dx.doi. org/10.1016/j.cplett.2009.09.064.
17. Carballeira, P. (2010). Mechanical and electrical properties of carbon nanofiber-ceramic nanoparticle-polymer composites (Tese de doutorado). Technischen Universit Kaiserslautern, Kaiserslautern.

18. Krause, B., Pötschke, P., \& Häußler, L. (2009). Influence of small scale melt mixing conditions on electrical resistivity of carbon nanotube-polyamide composites. Composites Science and Technology, 69(10), 1505-1515. http://dx.doi. org/10.1016/j.compscitech.2008.07.007.

19. Pötschke, P., Pegel, S., Claes, M., \& Bonduel, D. (2008). A Novel Strategy to Incorporate Carbon Nanotubes into Thermoplastic Matrices. Macromolecular Rapid Communications, 29(3), 244-251. http://dx.doi.org/10.1002/ marc. 200700637.

20. Díez-Pascual, A. M., Guan, J., Simard, B., \& Gómez-Fatou, M. A. (2012). Poly(phenylene sulphide) and poly(ether ether ketone) composites reinforced with single-walled carbon nanotube buckypaper: II - Mechanical properties, electrical and thermal conductivity. Composites. Part A: Applied Science and Manufacturing, 43(6), 1007-1015. http://dx.doi. org/10.1016/j.compositesa.2011.11.003.

21. Li, L., Li, B., Hood, M. A., \& Li, C. Y. (2009). Carbon nanotube induced polymer crystallization: The formation of nanohybrid shish-kebabs. Polymer, 50(4), 953-965. http:// dx.doi.org/10.1016/j.polymer.2008.12.031.

22. Kim, J. Y., Park, H. S., \& Kim, S. H. (2006). Unique nucleation of multi-walled carbon nanotube and poly(ethylene 2,6-naphthalate) nanocomposites during non-isothermal crystallization. Polymer, 47(4), 1379-1389. http://dx.doi. org/10.1016/j.polymer.2005.12.042.

23. Díez-Pascual, A. M., Ashrafi, B., Naffakh, M., GonzálezDomínguez, J. M., Johnston, A., Simard, B., Martinez, M. T., \& Gómez-Fatou, M. A. (2011). Influence of carbon nanotubes on the thermal, electrical and mechanical properties of poly(ether ether ketone)/glass fiber laminates. Carbon, 49(8), 2817-2833. http://dx.doi.org/10.1016/j.carbon.2011.03.011.

24. Wu, D., Wu, L., Yu, G., Xu, B., \& Zhang, M. (2008). Crystallization and thermal behavior of multiwalled carbon nanotube/poly(butylenes terephthalate) composites. Polymer Engineering and Science, 48(6), 1057-1067. http://dx.doi. org/10.1002/pen.21049.

25. Chen, E. C., \& Wu, T. M. (2008). Isothermal and nonisothermal crystallization kinetics of nylon 6/functionalized multi-walled carbon nanotube composites. Journal of Polymer Science. Part B, Polymer Physics, 46(2), 158-169. http://dx.doi.org/10.1002/ polb.21351.

26. Wang, B., Sun, G., Liu, J., He, X., \& Li, J. (2006). Crystallization behavior of carbon nanotubes-filled polyamide 1010. Journal of Applied Polymer Science, 100(5), 3794-3800. http://dx.doi. org/10.1002/app.23805.

27. Wu, D., Wu, L., Zhou, W., Yang, T., \& Zhang, M. (2009). Study on physical properties of multiwalled carbon nanotube/ poly(phenylene sulfide) composites. Polymer Engineering and Science, 49(9), 1727-1735. http://dx.doi.org/10.1002/ pen. 21403 .

28. Díez-Pascual, A. M., Naffakh, M., Marco, C., \& Ellis, G. (2012). Mechanical and electrical properties of carbon nanotube/poly(phenylene sulphide) composites incorporating polyetherimide and inorganic fullerene-like nanoparticles. Composites. Part A, Applied Science and Manufacturing, 43(4), 603-612. http://dx.doi.org/10.1016/j. compositesa.2011.12.026.

29. Levon, K., Margolina, A., \& Pathashinsky, A. Z. (1993). Multiple percolation in conducting polymer blends. Macromolecules, 26(15), 4061-4063. http://dx.doi. org/10.1021/ma00067a054 
30. Dai, K., Xu, X.-B., \& Li, Z.-M. (2007). Electrically conductive carbon black (CB) filled in situ microfibrillar poly(ethylene terephthalate) (PET)/polyethylene (PE) composite with a selective CB distribution. Polymer, 48(3), 849-859. http:// dx.doi.org/10.1016/j.polymer.2006.12.026.

31. Noll,A., \& Burkhart, T. (2011). Morphological characterization and modelling of electrical conductivity of multi-walled carbon nanotube/poly(p-phenylene sulfide) nanocomposites obtained by twin screw extrusion. Composites Science and Technology, 71(4), 499-505. http://dx.doi.org/10.1016/j. compscitech.2010.12.026.

32. Han, M. S., Lee, Y. K., Lee, H. S., Yun, C. H., \& Kim, W. N. (2009). Electrical, morphological and rheological properties of carbon nanotube composites with polyethylene and poly(phenylene sulfide) by melt mixing. Chemical Engineering Science, 64(22), 4649-4656. http://dx.doi. org/10.1016/j.ces.2009.02.026.

33. Yang, J., Xu, T., Lu, A., Zhang, Q., \& Fu, Q.-J. (2008). Electrical properties of poly(phenylene sulfide)/multiwalled carbon nanotube composites prepared by simple mixing and compression. Applied Polymer Science, 109(2), 720-726. http://dx.doi.org/10.1002/app.28098.

34. Jang, Y. K., Jang, P. G., Kim, J. K., Park, M., \& Yoon, G. (2009). Electrical Properties of Imidazole-Modified MWNT/ Polyphenylenesulfide Composites Prepared by Melt Mixing. Journal of Nanoscience and Nanotechnology, 9(7), 4180-4186. http://dx.doi.org/10.1166/jnn.2009.M28.

35. Hardwick, D. P. A., Naylor, S. L., Bujkiewicz, S., Fromhold, T. M., Fowler, D., Patanè, A., Eaves, L., Krokhin, A. A., Wilkinson, P. B., Henini, M., \& Sheard, F. W. (2006). Effect of inter-miniband tunneling on current resonances due to the formation of stochastic conduction networks in superlattices. Physica E, Low-Dimensional Systems and Nanostructures, 32(1-2), 285-288. http://dx.doi.org/10.1016/j. physe.2005.12.054.

Enviado: Nov. 11, 2013

Reenviado: Jul. 04, 2014

Aceito: Ago. 04, 2014 